\title{
Adquisición de morfemas del español
}

\section{Miguel Pérez Pereira*}

Universidad de Santiago

Dolores Singer

\section{INTRODUCCION}

El intcrés por ver cómo los niños adquieren las «unidades mínimas con significación lingüística», cuyo uso está regulado por reglas, viene ya desde antiguo. Los primeros estudios especificos sobre el tema consistieron en observaciones no sistemáticas sobre el uso de ciertos morfemas en niños (Guillaume, 1927; Smith, 1933; Ervin, 1964; Leopold, 1949; Menyuk, 1969; Ervin y Miller, 1964; Brown y Fraser, 1964). En estos primeros trabajos se resaltó el llamado fenómeno de la sobrerregularización, o uso excesivamente amplio de ciertas reglas de formación de formas regulares (plurales, pasados...), que se aplican indebidamente a formas irregulares. Ejemplos de este fenómeno son los típicos casos del inglés, "mans" en vez de "men», "foots" en vez de "feet», "goed» en lugar de "went»; o del francés, "servis" por "sers", "couré» por "couru», etc. Este fenómeno mostraba claramente que incluso niños de menos de tres años empleaban reglas para formar ciertas formas gramaticales. Guillaume (1927) acuñó el término de construcciones por "analogía» que luego seria empleado por otros autores (Ervin, 1964; Rûke-Dravina, 1959) para explicar este fenómeno.

A partir de las investigaciones desarrolladas en la Universidad de Harvard, se realizaron una serie de trabajos basados en la recogida sistemática de muestras del lenguaje infantil en ambiente natural y caracterizados por el empleo de ciertos índices o criterios para juzgar cuándo se podia considerar que ya se había adquirido un cierto morfema. Estos estudios eran tanto longitudinales (Cazden, 1968; Brown, 1973; Kuczaj II, 1977; James y Khan, 1982) como transversales (De Villiers y De Villiers, 1973, y Kuczaj II, 1977). Entre éstos destaca el ya citado de Brown (1973), que se convirtió en punto obligado de referencia de estudios posteriores. En todos estos estudios se pretendía establecer el orden en que se adquirian determinados morfemas del inglés.

Pero también en otras lenguas se han realizado estudios basados en observaciones sistemáticas del lenguaje infantil (Rûke-Dravina, 1959; Park, 1978; Mac Winney, 1976), algunos de los cuales presentan la 
ventaja de haber empleado la misma metodología y criterios que el de Brown (1973), con lo cual es más factible realizar comparaciones entre ellos.

A raiz del trabajo de Berko ( 1958 ) (coetáneo del del ruso Bogoyaulenskiy, 1957), se comenzó a emplear una técnica diferente para el estudio de la adquisición de los morfemas. Berko (1958) empleó, en un estudio experimental con grupos de sujetos de diferentes edades, palabras sin significado previo; con ello pretendia aislar el efecto de la práctica previa, o la familiaridad de uso, y así conocer mejor el empleo de reglas productivas de construcción de ciertas formas (plural, pasado de los verbos, presente progresivo, etc.).

Se han realizado posteriormente varias investigaciones que toman como base esta técnica (Anisfeld y Tucker, 1968; Derwing y Baker, 1977 y 1979). Este tipo de trabajos experimentales permite analizar con mayor precisión que los' estudios observacionales el tipo de reglas de construcción de morfemas que poseen los niños en las diferentes etapas del desarrollo, asi como analizar el dominio que tienen en el uso de los diferentes alomorfos que presentan algunos morfemas.

A pesar de esta larga y prolífica tradición en el estudio de la adquisición de morfemas, no tenemos conocimiento de que se haya llevado a cabo alguno con niños hispano-parlantes. Esto se hace más necesario, si cabe, si tenemos en cuenta la diversidad de recursos que existen entre distintas lenguas a la hora de modular los significados de las raíces de las palabras, y construir las formas que corresponden a los diferentes morfemas gramaticales. De ahi que no se puedan trasplantar mecánicamente los resultados hallados en una lengua a los de otra. Un ejemplo claro de lo que decimos lo tenemos en la expresión del tiempo pasado en inglés y en español, en el que es notablemente más compleja.

En la presente investigación se trata de estudiar la evolución que se da en la adquisición de morfemas y uso de reglas morfológicas para la construcción del plural, pretérito indefinido, pretérito imperfecto de indicativo, gerundio, aumentativo y diminutivo, en niños españoles.

\section{METODOLOGIA}

Material.-Se aplicaron a los sujetos dos tipos de pruebas de elicitación de las formas estudiadas. En las primeras, los niños debian realizar sus construcciones sobre palabras con sentido (P.R. $=$ palabras reales) y ya conocidas por ellos. En las segundas, debían realizarlas sobre palabras nuevas, inventadas por los experimentadores siguiendo las reglas fonológicas de la lengua y que, obviamente no poseían sentido previo (P.A. = palabras artificiales).

Tanto un tipo como otro de palabras fueron presentadas a los niños en unas cartulinas con dibujos brillantemente coloreados, que en el caso de las palabras reales representaban el objeto, cosa o acción, al que la palabra hacia referencia; y en el caso de las palabras sin sentido, simbolizaban objetos, cosas o acciones imaginarias o inventadas, las cuales pretendian representar a la palabra artificial.

El material empleado para el estudio del uso productivo de los 206 morfemas fue de 29 palabras reales y 31 palabras artificiales. Se 
pretendió cubrir el espectro de uso de los diferentes alomorfos de cada morfema. La lista de todas ellas es la que sigue.

\section{Plural}

Palabras reales: -alomorfo, -s (3): naranja, globo, cohete; alomorfo, -es (4): flor, árbol, patín, autobús; alomorfo, -o (1): paraguas.

Palabras artificiales: alomorfo, -s (4): pátula, estipa, lando, tapo; alomorfo -es (4): sibil, tipón, astor, patús.

\section{Diminutivo}

Palabras reales (3): árbol, jirafa, palmera.

Palabras artificiales (4): pátula, patús, estipa, astor.

\section{Aumentativo}

Palabras reales (3): botella, pelota, perro.

Palabras artificiales (4): sibil, tipón, lando, tapo.

Para cada uno de los morfemas verbales de gerundio, pretérito imperfecto y pretérito indefinido, se emplearon los siguientes verbos:

Palabras reales, 1.a conjugación ( $s$ ): dibujar, llorar, bailar, jugar, andar; $2 .^{\mathrm{a}}$ conjugación $(s)$ : beber, leer, comer, recoger, traer; $3 .^{\mathrm{a}}$ conjugación (s): dormir, abrir, conducir, oir, escribir.

Algunos de estos verbos presentaban formas irregulares para algunos de sus tiempos: andar (indefinido, anduvo); leer (gerundio, leyendo; indefinido, leyó); traer (indefinido, trajo; gerundio, trayendo; dormir (gerundio, durmiendo; indefinido, durmió) y oír (gerundio, oyendo; indefinido, oyó).

Palabras artificiales: $\mathrm{I}^{\mathrm{a}}$ conjugación $(\mathrm{s})$ : autar, driar, pondar, espidar, remogar; 2.a conjugación ( $s$ ): esmecer, cober, llecer, crocer, taer; $3 .^{\text {a }}$ conjugación ( $s$ ): dremir, ballir, ducir, oscluir, albucir.

Obviamente, entre estos no hay irregulares.

\section{Sujetos}

Inicialmente, se pretendió emplear grupos de edad de $\mathrm{N}=30$ sujetos, con igual número de niños que de niñas. Las dificultades halladas para la aplicación de la prueba a los sujetos más pequeños (tres años), nos obligaron a utilizar un número menor de sujetos en este grupo $(\mathrm{N}=19)$. (Es de resaltar que en ninguna investigación anterior se señala esta dificultad por parte de los niños de tres años).

Asi, pues, hubo tres grupos de cuatro, cinco y seis años, de 30 sujetos cada uno, y un grupo de edad de tres años, con 19 sujetos, de los cuales 1 I eran niñas y 8 niños. En total se aplicó la prueba a rog 
sujetos, entre tres y seis años, ambas edades inclusive, y medias de 3 , $3,4,8,5,8$ y 6,3 .

Los sujetos fueron escogidos de la población escolar y preescolar de La Coruña. Todos eran monolingües hablantes del castellano y de un medio socio-económico medio-alto.

\section{Procedimiento}

Cada palabra fue introducida en un texto en el cual se omitia la forma que se pretendia elicitar en el niño. Asi, por ejemplo, el tipo de texto para el morfema de plural era: «Esto es un globo». «Ahora hemos puesto otro más. Ahora hay dos___. Para la elicitación del diminutivo se empleaba el siguiente texto: «Esta es una pátula grande. Es una patulaza. Esta otra es una pátula pequeña, es una___" Para el aumentativo se empleaba la siguiente consigna: "Este es un sibil pequeño. Es un silibito. Este otro es un sibil grande. Es un __" " Para elicitar el morfema de gerundio se empleaba el siguiente tipo de texto: “Este niño sabe dibujar. El lo está haciendo ahora. ¿Qué está haciendo él ahora? El ahora está__. Para la elicitación del pretérito imperfecto de indicativo y del pretérito indefinido se emplearon respectivamente las siguientes consignas: "Este estipa sabe autar. El también lo hacía antes. ¿Qué hacia él antes? El antes___", y «Este estipa sabe autar. El también lo hizo ayer. ¿Qué hizo él ayer? El ayer - ${ }^{\prime \prime}$.

Estas consignas se presentaban simultáneamente con sus correspondientes cartulinas. Previamente a cada prueba con cada morfema se presentaban dos ejemplos a fin de asegurarnos que el niño la entendía.

Las pruebas se aplicaron individualmente a cada niño. Durando cada aplicación entre quince y treinta minutos.

Como se puede apreciar, en general, la técnica empleada es semejante a la ya usada por Berko (1998). El aspecto más novedoso es la presentación de items con palabras reales, y la mayor amplitud de la muestra de palabras que usan los diferentes morfemas. Si un niño sabe que el plural de árbol es «árboles» puede simplemente haber memorizado la forma del plural. Sin embargo, si él nos dice que el plural de una palabra artificial como «sibil» es «sibiles", tendremos evidencia de que está empleando una regla lingüística. La aplicación de reglas morfológicas a palabras artificiales demuestra que el niño sabe algo más que la palabra individual de su vocabulario; él posee reglas productivas aplicables a palabras nuevas. Se supone que, en general, los niños responderán mejor a los ítems con palabras réales, siendo la diferencia con los ítems con palabras artificiales achacable al efecto de la experiencia previa, o la familiaridad.

\section{RESULTADOS}

La exposición de los resultados se realizará por morfemas, presentando los resultados obtenidos en la prueba de palabras reales y en la 208 de palabras artificiales. Dado nuestro interés, no en cada item individual, sino en la clase a la que pertenecen, en las tablas, y gráficos que 
siguen se resumen las proporciones de aciertos por morfemas en cada alomorfo. Al no haberse encontrado diferencias significativas entre sexos en ninguno de los morfemas, los resultados se presentan sin diferenciar esta variable.

TABLA I

Morfema de Plural. Proporción de respuestas correctas

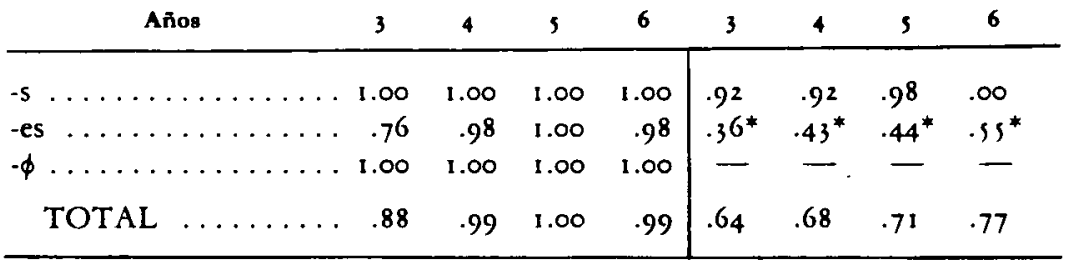

TABLA 2

Morfemas de Diminutivo y Aumentativo. Proporcion de respuestas correctas

P. $\mathbf{R}$.

P. A.

\begin{tabular}{ccccccccc} 
Años & \multicolumn{1}{c}{4} & 4 & $;$ & 6 & 3 & 4 & $;$ & 6 \\
\hline Diminutivo $\ldots \ldots \ldots \ldots$ & $.10^{*}$ & $.59^{*}$ & .83 & .90 & $.04^{*}$ & $.36^{*}$ & $.40^{*}$ & $.52^{*}$ \\
Aumentativo $\ldots \ldots \ldots \ldots$ & $.00^{*}$ & $.49^{*}$ & .69 & .83 & $.00^{*}$ & $.32^{*}$ & $.47^{*}$ & $.52^{*}$ \\
\hline
\end{tabular}

TABLA 3

Morfema de Gerundio. Proporción de respuestas correctas

P. R.

P. A.

\begin{tabular}{|c|c|c|c|c|c|c|c|c|}
\hline Años & 3 & 4 & 5 & 6 & 3 & 4 & 5 & 6 \\
\hline 1. ${ }^{2}$ conj. & .95 & 1.00 & 1.00 & 1.00 & $.57^{*}$ & .94 & .94 & .99 \\
\hline $2^{2}$ conj. . . . & .72 & .89 & .98 & .99 & $.33^{*}$ & $.56^{*}$ & $.59^{*}$ & .64 \\
\hline $3^{2}$ conj. . . . . . . & .66 & .85 & .90 & .94 & $.21^{*}$ & .63 & .74 & .79 \\
\hline TOTAL & .77 & .91 & .96 & .97 & $.37^{*}$ & .71 & .76 & .80 \\
\hline
\end{tabular}

TABLA 4

Morfema de Pretérito Imperfecto. Proporcion de respuestas correctas

P. R.

P. A.

\begin{tabular}{|c|c|c|c|c|c|c|c|c|}
\hline Años & 3 & 4. & 3 & 6 & 3 & 4 & 3 & 6 \\
\hline $1^{2}$ conj. & .72 & 1.00 & 1.00 & 1.00 & $.44^{*}$ & .97 & .98 & .97 \\
\hline $2^{2}$ conj. . . . . . & .60 & .94 & .95 & 1.00 & $.16^{*}$ & $.57^{*}$ & $.51^{*}$ & .68 \\
\hline $3^{2}$ conj. . . . . . . & .69 & .99 & .98 & 1.00 & $.29^{*}$ & .79 & .83 & .90 \\
\hline TOTAL & .65 & .98 & .98 & 1.00 & $.30^{*}$ & .77 & .78 & .84 \\
\hline
\end{tabular}

TABLA,

Morfema de Pretérito indefinido. Proporción de respuestas correctas

P. R.

P. A.

\begin{tabular}{|c|c|c|c|c|c|c|c|}
\hline Años & 4 & 5 & 6 & 3 & 4 & , & 6 \\
\hline$t^{2} \operatorname{conj} . \ldots \ldots \ldots .56^{*}$ & .76 & $.7^{6}$ & .78 & $.42^{*}$ & .92 & .93 & .96 \\
\hline 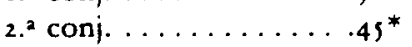 & .78 & .83 & .84 & $.21^{*}$ & $.37^{*}$ & $48^{*}$ & .62 \\
\hline 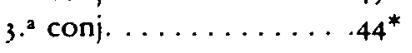 & .66 & .62 & .66 & $.31^{*}$ & .64 & .72 & .78 \\
\hline TOTAL & .74 & .73 & .76 & $.32^{*}$ & .64 & .71 & .78 \\
\hline
\end{tabular}


Los gráficos visualizan claramente que en todos los morfemas se aprecia una clara evolución (línea ascendente) en su adquisición, si exceptuamos aquellos casos (plural en -s, primera conjugación del gerundio en palabras reales) en que la prueba es ya realizada correctamente en su práctica totalidad, incluso por los sujetos más jóvenes. Esto revela que, evidentemente, durante el período evolutivo estudiado, se da un importante avance en el dominio de los morfemas estudiados.

Los datos nos indican también, inequivocamente, que las pruebas P. A. resultan siempre más dificiles que sus correspondientes P. R., pari todos los grupos de edad, lo cual revela el posible efecto

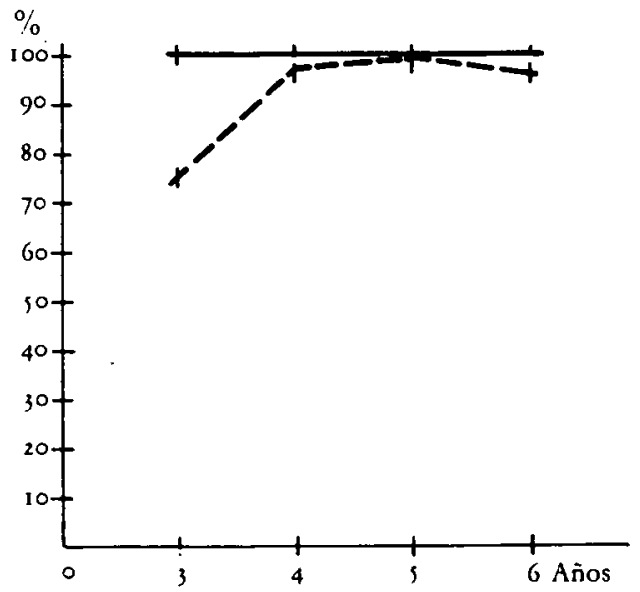

- Morfema. S. plural. P. R.

-1- Morfema. Es. plural. P. R.

Gráfico 1

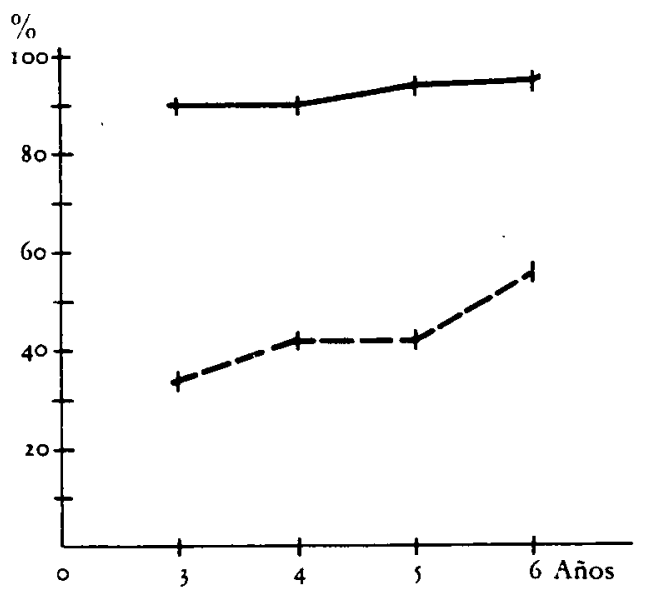

Morfema. S. plural. P. A.

- $\quad$ Morfema Es. plural. P. A.

Gráfico 2

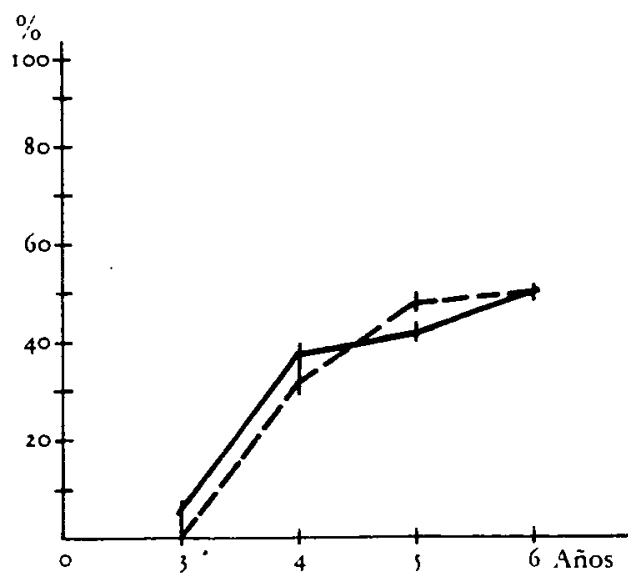

- - Aumentativo. P. A.

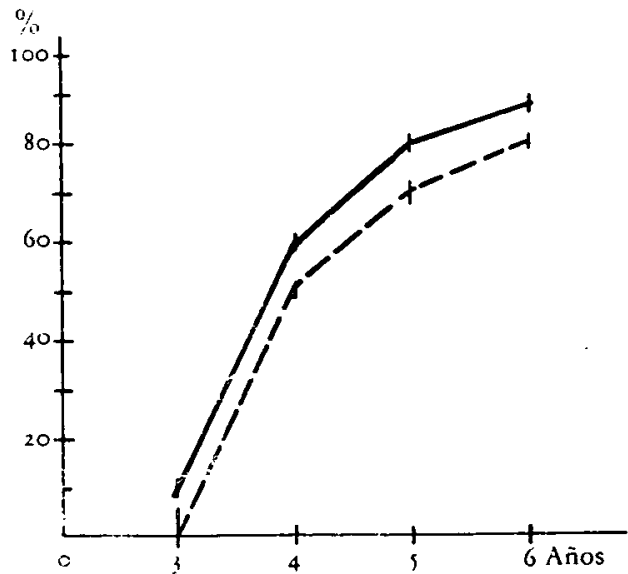

_.... Diminutivo. P. K.

Aumentativo P R. 

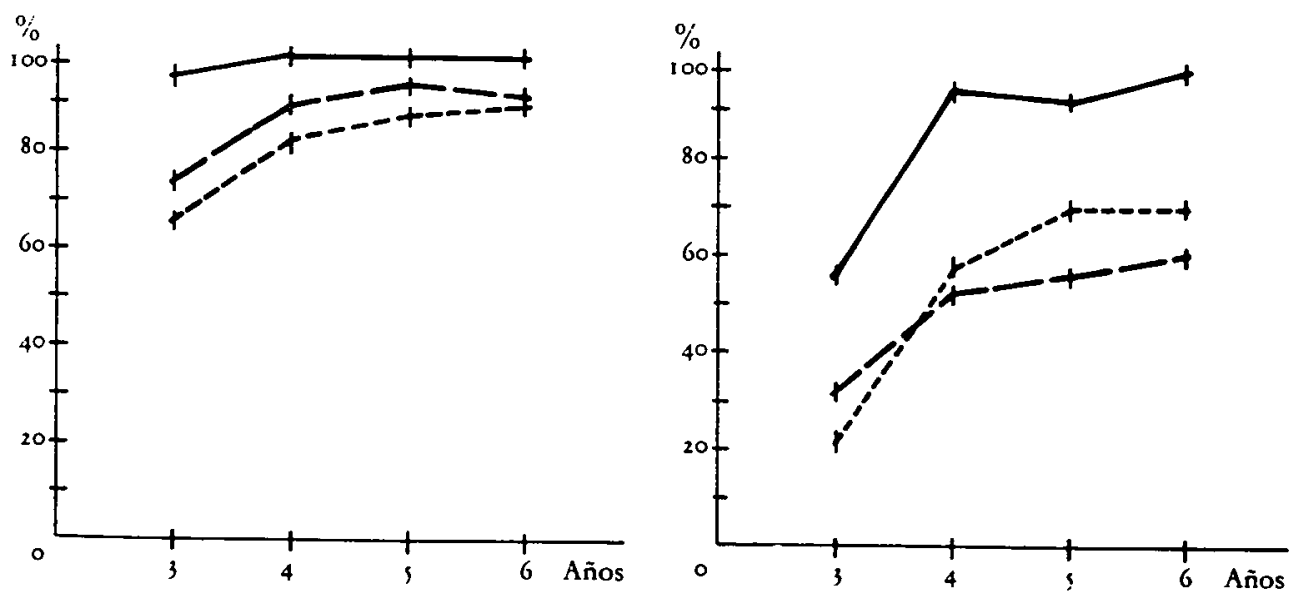

— I.2 Conjugación. Gerundio. P. R.

- 2.2 Conjugación. Gerundio. P. R.

3.2 Conjugación. Gerundio. P. R.

Gráfico ,

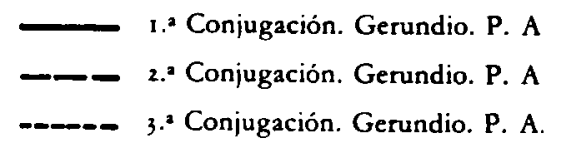

Gráfico 6

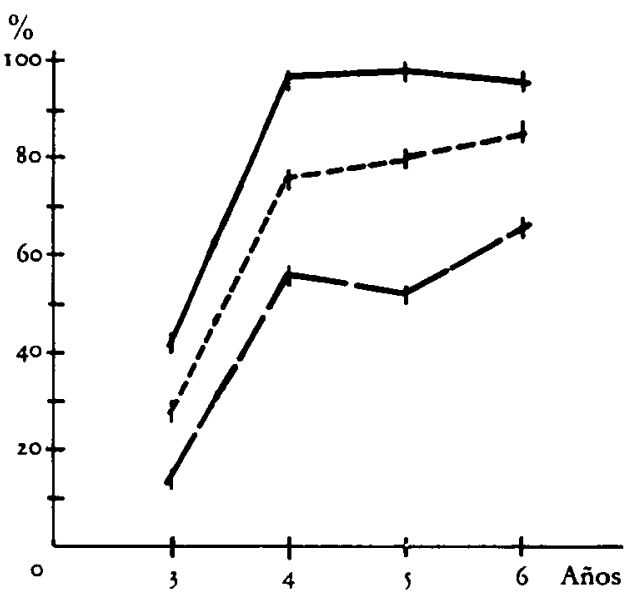

- 1. Conjugación. Imperfecto. P. A

- 2.2 Conjugación. Imperfecto. P. A.

- $3 .^{2}$ Conjugación. Imperfecto. P. A.

Gráfico 8

facilitador de la experiencia previa o familiaridad en la realización de las pruebas con palabras reales, tal como también había hallado Ervin (1964). Las diferencias halladas son, en general, significativas estadísticamente $(A<0.264$, aplicando la prueba $A$ de Sandler, para un nivel de significación de 6,05 y 29 g.l. (Seoane, i 980), excepto en el caso de los plurales en -s paza todos los niveles de edad, aumertativos y diminutivos en el grupo de tres años, primera conjugación del pretérito imperfecto en los grupos de cuatro, cinco y seis años, indefinido (total) en los grupos de cinco y seis años, y tercera conjugación del indefinido en los sujetos de cuatro años. 


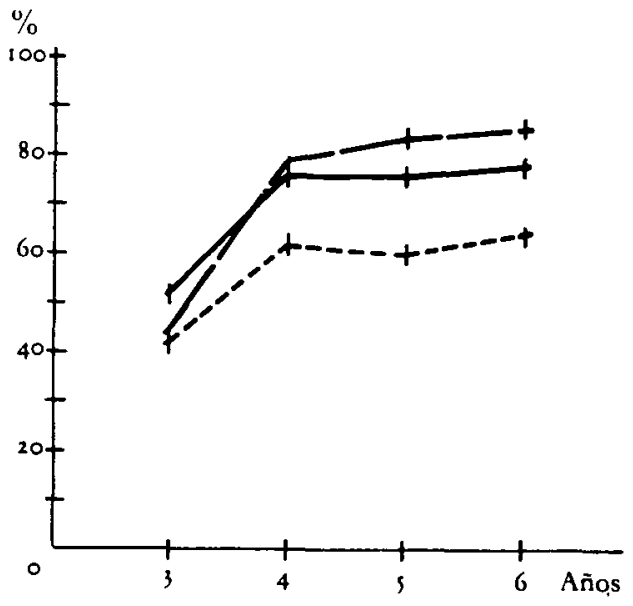

I.2 Conjugación. Indefinido. P. R. $-2 .^{2}$ Conjugación. Indefinido. P. R. $33^{2}$ Conjugación. Indefinido. P. R. Gráfico 9

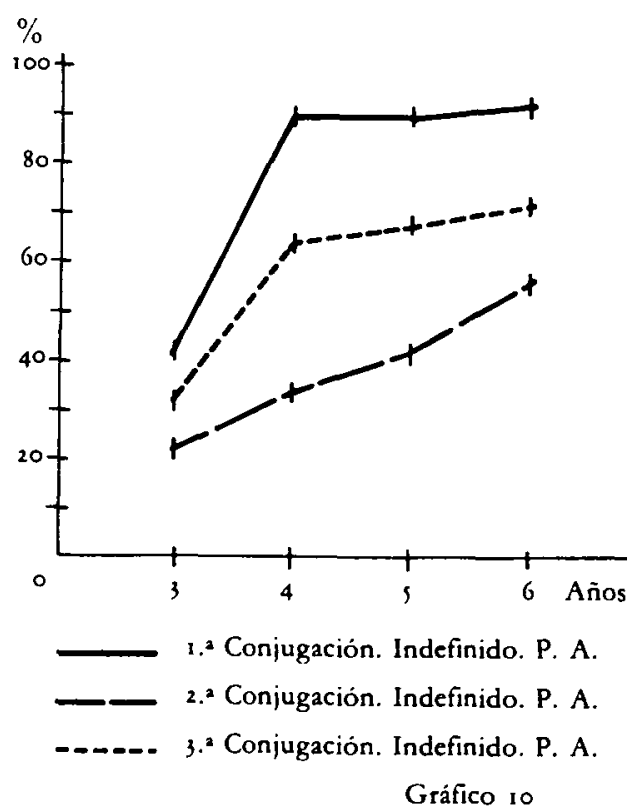

Gráfico io

La única excepción a esta pauta general de mayor facilidad de las pruebas P. R., se halla en el caso del morfema de pretérito indefinido en los verbos de la $1 .^{a}$ y $3 .^{a}$ conjugación, y especialmente con los sujetos más mayores. Aquí, sin duda, afectó a los resultados el hecho de emplear en la prueba P. R. verbos (andar, traer, conducir, dormir) que forman este tiempo de una manera irregular (anduvo, trajo, condujo, durmió); obviamente esta dificultad no existe tratándose de palabras artificiales. Esto indica la dificultad que tienen los niños en formar los pretéritos indefinidos irregulares, como veremos en el análisis de los errores cometidos.

La aplicación de la prueba estadística de comprobación de hipótesis para una sola proporción (Amón, 1982), nos permitió establecer si los sujetos de cada grupo habían llegado a unos resultados que significaban haber alcanzado un nivel de adquisición de las habilidades que exigía cada prueba, o, por el contrario, no. En los cuadros de proporciones de aciertos constan con un asterisco aquellas que no alcanzan el nivel de adquisición (valores $z$ comprendidos entre $+1: 96$ y $-1: 97$, para un nivel de significación de 0.05 ).

Anteriormente hemos apuntado una mejora progresiva en la realización de las pruebas a medida que aumenta la edad de los sujetos. En algunos casos las diferencias halladas entre grupos de edad en la realización de una misma prueba han sido significativas (prueba estadistica de comprobación de hipótesis para dos proporciones con observaciones independientes: Amón, 1982), indicando un salto importante en el proceso de adquisición de los morfemas.

En las pruebas $P$. R. se han hallado diferencias significativas $(z> \pm 2.58$, para un nivel de significación de 0.01 ) entre los resultados del grupo de tres y de cinco años en plurales acabados en -es; en diminutivos, entre el grupo de tres años, y los de cuatro, cinco y seis; además en esta prueba también se ha hallado diferencia significativa 
entre el grupo de seis años y el de cuatro. En aumentativos se ha encontrado diferencia significativa entre el grupo de tres años, y los de cuatro, cinco y seis; también la ha habido entre el de seis y el de cuatro años. En la prueba correspondiente a la segunda conjugación del gerundio, ha habido diferencias entre el grupo de cinco años, y el de tres. En el conjunto de las pruebas para elicitar los morfemas de imperfecto, se encontró diferencia significativa entre el grupo de tres años, y los de cuatro, cinco y seis años; esto mismo se halló en cada una de las tres conjugaciones por separado. En las pruebas de indefinido se halló diferencia significativa en la $2 .^{3}$ conjugación, entre el grupo de tres años, y los de cinco y seis.

En las pruebas P. A. se ha encontrado diferencia significativa en diminutivos, entre los niños de tres años, y los de cuatro, cinco y seis. En el gerundio, globalmente, entre los tres años, y los cinco y seis; además se ha hallado diferencia significativa también entre los grupos de seis, cinco y cuatro años, y el de tres, en la $\mathrm{r}^{\mathrm{a}}$ y $3 .^{\mathrm{a}}$ conjugaciones. En el imperfecto, globalmente, entre los grupos de seis, cinco y cuatro años, y el de tres, al igual que en la $1 .^{\text {a }}$ y $3 .^{2}$ conjugaciones; en la $2 .^{\text {a }}$, entre los resultados del grupo de tres años, y los de cuatro y seis. En el indefinido, teniendo en cuenta los resultados de las tres conjugaciones globalmente, entre los grupos de seis y cinco años, y el de tres; en la I $^{a}$ conjugación, entre el de tres, y los de seis, cinco y cuatro; en la $2 .^{2}$, entre el de tres, y el de seis; y en la $3 .^{a}$, entre el de tres, y los de seis y cinco años.

Estos resultados indican la existencia de un salto importante, en el proceso de adquisición de los morfemas, entre los tres años y los posteriores, manteniéndose después, entre cuatro y seis años, un ascenso gradual más suave.

De esta tónica general de desarrollo, cabe destacar los enormes progresos que se advierten en las pruebas de diminutivos, aumentativos, imperfecto, y 2.a conjugación del indefinido, en palabras reales; y en las pruebas de diminutivos, aumentativos, gerundio en la . $^{\text {a }} \mathrm{y}$ $3 .^{\mathrm{a}}$ conjugación, indefinido, e imperfecto, en las pruebas con palabras artificiales. En las pruebas de plural es en las que se aprecia, sin lugar a dudas, menos mejora en la realización.

Los resultados del grupo de cuatro años parecen asemejarse más a los de los grupos de cinco y seis, que a los resultados del grupo de niños de tres años:

Las pruebas en las que no se encontraron diferencias significativas en la realización entre grupos de edades diferentes, suelen ser pruebas en las que todos los sujetos muestran un alto nivel de aciertos (plurales en $-s$, gerundio de la $\mathrm{I}^{\mathrm{a}}$ conjugación en P. R.). En otros casos - como los del indefinido de la 1 . $^{\mathrm{a}}$ y $3 .^{\mathrm{a}}$ conjugación en $\mathrm{P}$. R., o plurales en -es en P. A. - la no existencia de diferencias significativas entre los sujetos más pequeños y los de más edad, parece deberse a la no superación, por parte de éstos últimos, de ciertas dificultades que le permitan un dominio de la prueba, y un despegue de los de menos edad, como si todavia se encontrasen en un nivel de transición hacia uno de mayor dominio.

Teniendo en cuenta los resultados reflejados en los cuadros, se puede establecer un orden de dificultad entre las pruebas, para cada uno de los diferentes niveles de edad. 
Destaca la enorme dificultad relativa que presentan los niños más pequeños (especialmente los de tres años) en la correcta formación de los diminutivos ! los aumentativos. Esto parece algo paradójico, al considerarse tradicionalmente que el habla de los niños más pequeños se caracteriza por el amplio empleo de diminutivos-afectivos, especialmente. Los resultados de las pruebas contradicen esta idea. Podria caber tarnbién la posibilidad de que las pruebas para estos dos morfemas no sean adecuadas, tal como ya apuntaron Derwing y Baker (1977, 1979). Nosotros, no obstante, no participamos de esta opinión. El incremento progresivo en la proporción de aciertos apunta nuestro criterio y parece que las dificultades de los niños de tres años son reales, y no por inadecuación de la prueba.

También destaca la mejora progresiva, en relación a otras pruebas, en el imperfecto, que pasa de ser la prueba tercera mejor realizada a los tres años (.65) a ser la primera mejor realizada a los seis años ( 1.00 ). En esta prueba, junto con la de los aumentativos y diminutivos y la del indefinido, es en la que se aprecia un progreso evolutivo de mayor magnitud, si bien el punto de partida era más elevado, de ahi su progreso importantc en el orden, $y$ que se apreciasen diferencias significativas claras entre grupos de edad, como vimos anteriormente.

Fn todos los grupos de edad son las pruebas mejor realizadas las del plural, gerundio e imperfecto, si bien hay cambios en su orden de dificultad.

En los sujetos de cuatro, cinco y seis anoos se mantiene el mismo grado de dificultad en las diferentes conjugaciones de gerundio ( $\mathbf{r}^{\mathrm{a}}$ conjugación $<2 a^{\mathrm{a}}$ conjugación $<3 .{ }^{\mathrm{a}}$ conjugación) y de indefinido (2.a conjugación $<1$. $^{\mathrm{a}}$ conjugación $<3$. $^{\mathrm{a}}$ conjugación).

En las pruebas de palabras artificiales se aprecia también una dificultad importante en la realización de las de aumentativos $y$ diminutivos, que ahora se extiende a todas las edades (en ninguna se logra el nivel de adquisición).

Es también realmente sorprendente la dificultad de los sujetos con la prueba de plurales en -es, que hace que la prueba global de plurales vaya ocupando progresivamente un lugar más bajo en el orden de realización, pasando del primero a los tres años, al cuarto a los seis, sólo por encima de aumentativos y diminutivos.

En todos los grupos de edad se aprecia que, tanto en el gerundio como en el imperfecto como en el indefinido, los niños realizan mejor siempre la primera conjugación, después la tercera y finalmente la segunda. La única excepción es la de los sujetos de tres años con el gerundio.

\section{ANALISIS DE LOS ERRORES}

E.l análisis de los errores cometidos por los niños es un valioso elemento para conocer mejor el tipo de reglas que emplean en cada edad o el grado de desarrollo en el dominio del uso de los morfemas.

En el caso de los plurales, únicamente es provechoso el análisis de los errores cometidos en la prueba de plurales en -es, en palabras reales 
y en palabras sin sentido. Tanto en un caso como en otro, los errores cometidos más frecuentemente consistieron en no añadir ningún sufijo a la palabra y dejarla tal como está, o añadirle el sufijo -s a palabras terminadas en consonante y que requeririan el sufijo -es para ser pluralizadas. Estos dos tipos de errores se reparten casi por igual el total de errores cometidos y son producidos por los niños de todas las edades. En el caso de la prueba con palabras artificiales, destacan los errores cometidos con la palabra "patús», que no fue pluralizada en ningún caso por los sujetos de cuatro y cinco años, y sólo en una proporción de .07 y .16 por los de seis y tres años, respectivamente. Parece en éste caso que los niños actúan como si «patús», que termina en -s, fuese ya una palabra que se encuentra en su forma plural. No obstante, en palabras reales (P. R.) realizaban correctamente la diferencia entre "paraguas» (plural "paraguas») y «autobús» (plural «autobuses"). Sin duda, la existencia en español de palabras terminadas en -s que pluralizan con el alomorfo $-\phi$ dificulta la realización en esta palabra.

En el caso de las palabras reales «paraguas» y «autobús», la familiaridad de estas palabras debe tener un efecto positivo sobre los resultados.

En las pruebas de elicitación de aumentativos y diminutivos con palabras reales y con palabras artificiales, sí que se aprecia, por el contrario, alguna diferencia. Mientras que con palabras reales los niños emplean exclusivamente los sufijos -ito, -ita (según el género de la palabra), para formar los diminutivos, y -azo, -aza, para formar los aumentativos; con palabras artificiales emplean también en algunos casos el sufijo -in para los diminutivos y el sufijo -on, -ona (según el género de la palabra) para los aumentativos, pero en una proporción mayor (casi la mitad de las respuestas).

Los niños de cinco y sèis años cometieron sobre todo errores que consistian en añadir un sufijo correcto, pero modificando la palabra original (ej. de "árbol", "arbito»; de «botella», "botaza» o «brotaza»; de "perro", "perrotazo", o en P. A. de "sibil", "sibazo"), y en modificar la palabra original añadiéndole un sufijo incorrecto, cosa que sólo se hacia con los aumentativos én el caso de P. R. (ej. de "botella», "botelleza»). Con palabras artificiales también añadian un sufijo erróneo a la palabra no modificada. Los niños pequeños presentaron, sobre todo, errores consistentes en no añadir nada a la palabra ofrecida, repitiéndola igual, y también, pero en menor cuantia, errores consistentes en añadir a la palabra un adjetivo, que en el caso de los diminutivos era "pequeño" y en el de los aumentativos "grande».

Este último tipo de error, a diferencia de los demás, se dio exclusivamente en los sujetos más pequeños (tres y cuatro años), siendo su frecuencia mayor a los tres años, tanto en palabras reales como en palabras artificiales. Dicho error también fue hallado en otras investigaciones (Berko, 1958), aún en mayor proporción.

Una palabra con la que todos los sujetos produjeron una gran cantidad de errores en la prueba de aumentativo fue "tipón" (artificial). La proporción de respuestas erróneas osciló entre .70 y 1.00, según las edades, y la inmensa mayoría consistieron en no añadir nada a la palabra. Este comportamiento probablemente sea debido a que su terminación en -ón puede confundirla con un aumentativo. 
En los morfemas verbales se aprecian unos comportamientos constantes a través de cada uno de ellos, con diferentes comportamientos para las P. R. y las P. A., y con diferencias claras entre el grupo de niños de tres años y los demás.

Con P. R. la inmensa mayoría de los errores que cometen los niños de cuatro, cinco, y seis años se dan en verbos que forman el gerundio o el indefinido de una manera irregular. Los niños de tres años, además de cometer este tipo de errores en una proporción elevada, dan con más frecuencia aún errores consistentes en no añadir ninguna desinencia y repetir la palabra tal como se le presentó.

Los errores comeridos con estos verbos irreguiares son de sobrerregularización (Dale, 1976) y consisten en conjugarlos como si fuesen un verbo regular.

En el gerundio destacan los errores cometidos con los verbos «leer» (leyendo), "traer» (trayendo), "dormir" (durmiendo) y "oir" (oyendo). En los casos de "leer» y «traer» ( $2 .^{2}$ conjugación), los errores consistieron en añadir una desinencia incorrecta (-indo, o -endo) a la raíz del verbo (ej. traíndo, leendo). En esta segunda conjugación también se cometieron errores de esta misma índole con el verbo «beber» (bebendo), que es regular.

En los casos de "dormir» y "oír» ( $3^{a}$ conjugación), los niños producian "dormiendo", sin cambiar la vocal del radical, y uoindo", añadiendo incorrectamente la deșinencia -indo.

En el imperfecto, al no existir verbos que lo formen irregularmente, los sujetos dan muchos menos errores ${ }^{1}$. Sólo cabe destacar los de los niños de tres años, que consisten, otra vez, en no añadir nada al verbo presentado, o en añadir formas de otros tiempos (principalmente del gerundio).

En el indefinido se produce la misma pauta de respuestas. Los niños de cuatro, cinco y seis años cometen en su mayoría errores de "sobrerregularización» (.16, .19, .17 y .13, para la primera conjugación; .15, .1 $5, .18, .15$, para la segunda, y .31,.33,.31 y .23, en la tercera, para seis, cinco, cuatro y tres años, respectivamente), mientras que los de tres años, aun cometiendo muchos errores de sobrerregularización con los verbos irregulares, cometen más errores consistentes en no añadir sufijo a la forma verbal dada y repetirla tal cual $(.19, .28, .13$, para la $1 .^{2}, 2 .^{a}$ y $3 .^{a}$ conjugación, respectivamente).

En la $1^{a}$ conjugación, los sujetos fracasan especialmente con el verbo "andar", que foima de una manera irregular su indefinido (anduvo) $(.96, .93, .96,1.00$, de proporción de respuestas erróneas para seis, cinco, cuatro y tres años; respectivamente). Con este verbo los niños produjeron como indefinido la forma "andó», un claro ejemplo de sobrerregularización de la desinencia -ó del indefinido regular de la $1 .^{2}$ conjugación.

En la $2 .^{a}$ conjugación, los errores de sobrerregularización se cometieron con el verbo "traer", que forma irregularmente su indefinido: "trajo»: La forma dada generalmente por los niños fue «trayón, si bien hubo algún caso esporádico de "trajió» o "traó», formas todas de transacción entre la raiz «tra-» y la desinencia «-ió», u «-ó». 
En la tercera conjugación los errores de sobrerregularización se cometieron con los verbos «dormir" y "conducir", que forman irregularmente su pretérito indefinido: «durmió» (con cambio en la vocal del radical) y "condujo». Sobre unas 3/4 partes de los errores de sobrerregularización corresponden al verbo «conducir». Los niños producen las. formas "dormió" y condució», claros ejemplos de aplicación de la desinencia regular -ió a la raíz invariada.

Además de estos errores, destacan también los cometidos con los irregulares «leer» (leyó), de la $2 .^{2}$, y «oír» (oyó), de la $3 .^{a}$ conjugación.

En lo que respecta a las pruebas con palabras artificiales (P. A.), se aprecian también pautas comunes en los errores cometidos por los niños con los gerundios, imperfectos e indefinidos.

Otra vez los errores cometidos mayoritariamente por los niños de tres años consisten en repetir el verbo tal como les fue enunciado, sin añadir a la raiz ninguna desinencia de gerundio, imperfecto o indefinido $\left(.40, .48, .60\right.$, para la $1 .^{a}, 2 .^{a}$ y $3 .^{a}$ conjugación del gerundio; $.52, .62$ y . 6 , para la $\mathrm{I}^{\mathrm{a}}, \mathrm{2}^{\mathrm{a}}$ y $3 .^{\mathrm{a}}$ conjugación del pretérito imperfecto de indicativo, y $39, .53$ y .54 , para la $1 .^{a}, 2 .^{a}$ y $3 .^{a}$ conjugación del pretérito indefinido, respectivamente).

Por su parte, los niños de cuatro, cinco y seis años apenas cometen errores en la $1^{a}$ conjugación de los tres tiempos, siendo su nivel de realización superior incluso al obtenido con palabras reales en la prueba de elicitación del pretérito indefinido.

En la $2 .^{2}$ y $3 .^{2}$ conjugación de los tres tiempos, el error que cometen mayoritariamente consiste en no modificar la raiz de la palabra ofrecida, pero añadir una desinencia incorrecta. Así, en la $2 .^{a}$ conjugación del gerundio añaden incorrectamente las terminaciones -endo (esmecendo, cobendo), o -ando $(.18, .19, .27$, para seis, cinco y cuatro años, respectivamente). Es de resaltar que los errores de por adición del sufijo -ando (que presentan proporciones de .05, .04 y .07, para cinco, cuatro y tres años, respectivamente) podrían ser debidos a un efecto de sobregeneralización a partir de la primera conjugación, cuya desinencia es -ando. En la $3 .^{\text {a }}$ conjugación, los sufijos empleados erróneamente son -endo, e -indo.

En el pretérito imperfecto, de los sufijos erróneos que solían añadir los niños a las raíces de los verbos (que constituian una proporción de $.13, .28$ y .22, para seis, cinco y cuatro años, en la $2 .^{\mathrm{a}}$ conjugación), sobre $1 / 3$ ó $3 / 4$ partes, según las edades, lo fueron de la $x^{a}$ conjugación -aba) (ejemplo, cobaba, de cober, crozaba, de crocer, llezaba, de llecer, en vez de cobia, crocía y llecía). Estos casos pueden ser ejemplo también de un efecto de sobregeneralización a partir de la primera conjugación.

Pero donde más claramente se aprecia este fenómeno de generalización errónea de la desinencia de la $1 .^{a}$ conjugación es en la $2 .^{a}$ y $3 .^{a}$ conjugaciones del pretérito indefinido.

Aquí la desinencia -ó de la $1^{2}$ conjugación se empleó en las proporciones del .18, .21, .36 y.14, en la 2.2, y .11, .06,.11, .04, en los verbos de la $3 .^{a}$ conjugación, para seis, cinco, cuatro y tres años, respectivamente. Los niños producen formas como «llezón, en vez de «lleció", "drumó», en vez de "drumió», "albuzó», en vez de "albució», etcétera. 
Otro fenómeno interesante a resaltar, pero ya más de tipo anecdótico, lo constituye lo que se podría denominar asimilación de un verbo artificial a un verbo real de forma parecida, a la hora de conjugarlo. Tenemos ejemplos de ello en la formación del gerundio con los verbos "espidar», "taer» y "oscluir», que aparecen como "espiando», del verbo "espiar»; "trayendo", de "traer", y «excluyendo», de «excluir». En la producción del imperfecto se formó «excluia» y en las del indefinido «excluó» y «excluyó», y «rrayó» y «traó». Obsérvese que, sobre todo en el indefinido, los niños cometen error, no obstante, en sus asimilaciones del verbo artificial al verbo real. Estos errores de asimilación los cometen especialmente los niños de cuatro, cinco y seis años, constituyendo a veces entre $1 / 2$ y $3 / 4$ del total de respuestas dadas a ese item.

\section{CONCLUSIONES}

Parece evidente, a la luz de los resultados obtenidos, que entre los tres y los seis años se produce un avance importante en el dominio de los morfemas estudiadus. En este proceso de adquisición se aprecia un salto notable entre los tres y los cuatro años, siendo el desarrollo posterior más gradual y menos brusco. Es en los morfemas de aumentativo, diminutivo, imperfecto $\mathrm{e}$ indefinido, en los que se aprecian mayores progresos.

En general se observa que para todos los niños las pruebas con palabras reales resultan más sencillas que las pruebas con palabras artificiales. Esto parece indicar, o bien que en la adquisición del lenguaje la familiaridad o frecuencia de uso parece jugar algún papel, o bien que, al igual que sucedió con los estudios de percepción del lenguaje (Garnica, 1973; Shvachkin, 1948), las situaciones artificiales confunden al niño, provocan una reacción de perplejidad y le impiden sostener el mismo comportamiento que con palabras y situaciones reales. Otra posibilidad es que exista un efecto combinado de estos aspectos.

Las formas más difíciles de adquirir parecen ser las de aumentativo 'y diminutivo y el plural en -es, que ni siquiera logran el nivel de adquisición a los seis años con P. A. Le siguen en dificultad de adquisición las segundas conjugaciones de los tres tiempos. El resto de las conjugaciones (si exceptuamos el problema de los irregulares en P. R.) se adquieren más fácilmente, siendo los plurales en -s (y en o en P. R.) los que se adquieren antes.

La pluralidad de formas que se emplean para derivar los aumentativos y los diminutivos es posible que dificulte su dominio, tal como se apreció que sucede con otros morfemas en otras lenguas (Park, 1978; Bogoyaulenskiy, 1957). No obstante, los resultados obtenidos por niños españoles son superiores a los hallados por Berko (1958) con niños norteamericanos.

En el caso de palabras que forman el plural en -es, los niños tienden a cometer errores consistentes en formar su plural con -s o no añadirle ningún sufijo. Por el contrario, las palabras que lo forman en -s son bien realizadas. Esto parece confirmar la idea ya anunciada por otros autores (Derwing y Baker, 1979), de la existencia de un período en que los niños parecen emplear un sistema de reglas de formación del plural 
que no abarca, en el caso del español a las palabras que lo hacen en -es, asimilándolas asi a las otras reglas productivas $(-5 \quad 0-\phi)$.

Hay dos factores que pueden determinar este comportamiento. Uno es la mayor simplicidad fonológica de los plurales en -s, que se adquieren antes. El otro es la mayor frecuencia en español de palabras que terminan en vocal y hacen el plural en -s (62 por 100 del total, según Navarro Tomás, 1966).

El tipo de errores cometidos también permite diferenciar nítidamente a los niños de tres años del resto, como ya hemos visto. Da la impresión de que los niños más pequeños todavía no manifiestan una productividad plena en la formación de palabras:

Los errores de sobrerregularización cometidos con los verbos irregulares reales, en la formación del gerundio y del indefinido, son teóricamente de gran importancia. Todos los niños manifiestan una gran dificultad con estos verbos, que lleva incluso a que la proporción de aciertos sea menor en pruebas de indefinido con $P$. R. que con $P$. A. Esto pone de manifiesto que si el proceso de adquisición de la morfología fuese el de memorización de casos aislados y no el de aprendizaje de reglas, el proceso sería mucho más lento.

Cuanta mayor simplicidad de formas y regularidad haya en la formación de morfemas, antes se dominarán éstos.

El fenómeno, también registrado con P. A., de sobregeneralización de los sufijos de la primera conjugación, para la formación del tiempo imperfecto e indefinido en las otras conjugaciones, puede ser interpretado también como una tendencia a buscar pautas generales de formación de los verbos, que no hace sino revelarnos el papel activo de los niños en el proceso de adquisición del lenguaje, y en su esfuerzo por buscar reglas productivas simples de formación de morfemas. Este esfuerzo, dada la complejidad de la lengua hace que cometan errores como los que acabamos de ver.

\section{Notas}

1 A pesar de todo, se han encontrado algunos errores que podrian ser explicados por una especie de "efecto de halo" de verbos que son irregulares en otros tiempos y que aumenta en algo la dificultad para producir sus imperfectos. Asi, encontramos formas como "trajia", "traeiba", "trajiendo", de "traer"; "dormión, "durmian, "dormiendo", de "dormir"; uleiba", de "leer", y upyá", "oyendo", de "oir".

\section{Resumen}

Se ban aplicado dos pruebas de palabras reales y palabras artificiales (semejante a la de Berko, 1958) a 109 niños bispano-parlantes de tres a seis años de edad, a fin de establecer la evolución en el uso de morfemas de plural, aumentativos y diminutivos, gerundio, pretérito imperfecto de indicativo y pretérito indefinido. Los resultados indican un progreso importante entre los tres y los cuatro años, siendo la curva posterior del desarrollo más suave. Se establece un orden de dificultad de estos morfemas, y se realiza un analisis de los errores cometidos, encontrándose también sobrerregularizaciones con verbos reales irregulares. Se extraen implicaciones acerca del proceso de adquisición de morfemas.

\section{Summary}

Proofs of real and artificial words (similar to Berko's, 19y8) were applied to rog spanish children from 3106 year-old, in order to determine the evolution in the use of plural morpbemes, augmentatives and diminutives, past imperfect, and past definite. Results show an important progress between, and 4 year-old, being the later growth smoother. It is established a difficulty order in the acquisition of these morpbemes, and errors made are analysed, being also find overregularization errors with irregular real verbs. Some implications were drawn on the morpbemes acquisition process. 


\section{Résumé}

On a appliqué deux epreuves de mots rêels et de mots artificiels (come celles de Berko, 1958) a des enfants de 3 à 6 ans s'exprimant en espagnol. Il s'agit d'ètablir l'evolution dans l'usage de morphèmes au pluriel, des augmentotifs et des diminutifs, du geroundif, du passé imparfait, et du passé simple. Les résultats indiquent un progrès important entre les 3 at les 4 ans, itant plus douce la progression posterieure. On a itabli aussi un ordre de difficulté dans lacquisition de ces morphemes et on a réalise une enalyse des erreurs commis. On a troule aussi des super-rigularisations avec des verbes réels irreguliers. On tire des implications à propos du procès d'acquisition de morphimes.

\section{Referencias}

AMON, J.: Estadistica para psicólogos. Madrid, Pirámide, 1982.

ANISFEI.D, M., \& TLCKER, R.: "English pluralization rules of six-year-old children. Child Detelopment, 1968, 38, págs. 1 201-1217.

BERKO, J.: "The child's learning of English morphology". Word, 1958, 14, págs. $190-177$.

Bogoyavi.esnkiY, D. N.: "The acquisition of Russian inflections". Ferguson, C. A.\&Slobin, D. I. (Eds.). Studies of cbild development. Nueva York, Holt, Rinehart \& Winston, 1973, págs. 284-292. (Original ruso, 1957).

BROWN, R: : A first language. Londres. George Allen and Unwin, 1973.

BROWN, R. y FRASER, C.: "The acquisition of syntax". En Bellugi, U. \& Brown, R. (Eds.). The acquisition of language. Monographs of the Society for Research in Child Development. 1964, 29 (1, Serial num. 92), págs 43-79.

CAZDEN, C. B.: "The acquisition of noun and verb inflections". Child Development. 1968, 39, págs. 433-438.

DAI,E, P.: Language, Development. Structure and Function. Nieva Yoik, Holt, Rinehart \& W'inston, 1970.

DERWING, B. L. y BAKER, W. J.: “The psychological basis for morphological rules». En MacNamara, J. (Eds.), Language, learning and thought. Nueva York. Academic Press, 1977, págs. 8 s-110.

DERW'ING, B. L., y BAKER, W. J.: "Recent research on the acquisition of English morphology", en Fi.etcher, P., y GARMAN, M. (Eds.), Studies in language acquisition. Cambridge University Press, I979, págs. $209-223$.

DF. Vil.liers, J. G., y DE Vil.t.ers, P. A.: "A cross-sectional study of the acquistion of grammatical morphemes in child speech". Journal of Psycholinguistic Research, 1973, 2, (3). Reproducido en BI.(O). J.. (Eds.), Readings in language development. Nueva York, John Wiley \& Sons, 1978, págs. 74-84.

ERviN, S. M.: “Imitación y cambio estructural en el lenguaje de los niños». En LENNEBERG, E. H., y otros, "Nuevas direcciones en el estudio del lenguaje", Revista de Occidente. Madrid, 1974, págs. 187-217. Original en inglés M. I. T. Press. Cambridge. Mass, 1964.

GARNIKA, O. K.: "The development of phonemic speech perception". En MOORE, T. (Ed.), Cognitive development and the acquisition of language. Nueva York, Academic Press, 1973, págs. $215-222$.

Gil.. GAYA, S.: Curso superior de sintaxis española. Barcelona. Vox, 1973.

GLil.t.Al:ME, P.: "Le développement des éléments formels dans le langage de l'enfant». Journal de psychologie. 1927, 24, págs. 203-229.

JAMES, S. L., y KHAN, L. M. L.: "Grammatical morpheme acquisition: an approximately invariant order?" Journal of Psycbolinguistic Research, 1982, $\mathrm{H}$, (4), págs. $381-388$.

KUCZAJ II, S. A.: "The acquisition of regular and irregular past tense forms". Journal of Verbol Learning and Verbal Behavior. 1977,16 , págs. $589-600$.

LEOPOI.D, W. F.: Speech development of a bilingual child: A linguist's record. Vol. III. Northwestern Univ. Press. Evanston. II 1,1949 .

LEVY, Y.: "The acquistion of Hebrew plurals: the case of the missing gender category". J. Child Lang., 1983,10 , págs. $107-121$.

MACWHINNEY, B.: "Hungarian research on the acquisition of morphology and syntax". Journal of Child Language. 1976, 3, págs. $397-410$.

MENYUK, P.: “Sentence children use. MIT Researcb Monograph. Núm. /2. M. 1. T. Press. Cambridge, I 972. MII.I.ER, W., y ERVIN, S.: "The development of grammar in child language». En BEI.I.LGi, U., y BROX'N, R. (Eds.): The acquisition of language, University of Chicago Press, 197\%, págs. 9-34.

Navarro TOMÁs, T.: Estudios de fonologia española. Las Américas Publishing Co. Nueva York, 1966.

PARK, T.: «Plurals in child speech". Journal of Child Language, 1978, , págs. 237-290.

Ruke-Dravina, V. (1973): "On the emergence of inflection in child language: A contibution based on Latuian speech data". En FERGUSON, C. A., y SLOBIN, D. I. (Eds.): Studies of child language development. Nueva York: Holt, Rinehart and Winston, 1973, págs. 252-267. Original publicado en 1959.

Seco, R.: Manual de gramática española. Madrid. Aguilar, 1973.

Seonne, J.: Psicologia matemática I. U. N. E. D. Madrid, 1980.

SHVACHKIN, N. K.: "The development of phonemic speech perception in early childhood". En FERGUSON, C. A., y Stobin, D. I. (Eds.): Siudies of child language development. Nueva York: Holt, Rinehart and Winston, 1973, págs. 91-123. Original en ruso, 1948 .

Slobin, D. I.: "On the learning of morphological rules: A reply to Palermo and Eberhard". En Si.oBin, D. I. (Eds.), The ontogenesis of grammar. Nueva York. Academic Press, 197t, págs. $215-223$.

Smith, M. E.: "Grammatical errors in the speech of preschool children». Child Development. 1933, 4. 183-190. Reimpreso en BI.OOM, L. (Ed.), Readings in Language Development. Nueva York, John Wiley \& Sons. 1978 , págs. $31-38$ 